\title{
Independent assessment of source position for gynecological applicator in high-dose-rate brachytherapy
}

\author{
Hiroyuki Okamoto, PhD', Satoshi Nakamura, Msc', Shie Nishioka, PhD', Kotaro lijima, Msc', Akihisa Wakita, Msc', \\ Yukinao Abe, Msc², Naoki Tohyama, Msc ${ }^{3}$, Shinji Kawamura, PhD', Toshiyuki Minemura, PhD 5 , Jun Itami, MD, PhD' \\ 'Department of Radiation Oncology, National Cancer Center Hospital, Tokyo, ²Department of Radiology, Chiba University Hospital, Chiba, \\ ${ }^{3}$ Division of Medical Physics, Tokyo Bay Advanced Imaging \& Radiation Oncology Clinic, Chiba, ${ }^{4}$ Department of Radiological Technology. \\ Faculty of Fukuoka Medical Technology, Teikyo University, Fukuoka, ${ }^{5}$ Center for Cancer Control and Information Services, National Cancer \\ Center, Tokyo, Japan
}

\begin{abstract}
Purpose: The aim of this study is to describe a phantom designed for independent examination of a source position in brachytherapy that is suitable for inclusion in an external auditing program.

Material and methods: We developed a phantom that has a special design and a simple mechanism, capable of firmly fixing a radiochromic film and tandem-ovoid applicators to assess discrepancies in source positions between the measurements and treatment planning system (TPS). Three tests were conducted: 1) reproducibility of the source positions $(n=5)$; 2 ) source movements inside the applicator tube; 3$)$ changing source position by changing curvature of the transfer tubes. In addition, as a trial study, the phantom was mailed to 12 institutions, and 23 trial data sets were examined. The source displacement $\Delta \mathrm{X}$ and $\Delta \mathrm{Y}$ (reference $=\mathrm{TPS}$ ) were expressed according to the coordinates, in which the positive direction on the $\mathrm{X}$-axis corresponds to the external side of the applicator perpendicular to source transfer direction Y-axis.

Results: Test 1: The $1 \sigma$ fell within $1 \mathrm{~mm}$ irrespective of the dwell positions. Test 2: $\Delta \mathrm{X}$ were greater around the tip of the applicator owing to the source cable. Test 3: All of the source position changes fell within $1 \mathrm{~mm}$. For postal audit, the mean and $1.96 \sigma$ in $\Delta \mathrm{X}$ were 0.8 and $0.8 \mathrm{~mm}$, respectively. Almost all data were located within a positive region along the $\mathrm{X}$-axis due to the source cable. The mean and 1.960 in $\Delta \mathrm{Y}$ were 0.3 and $1.6 \mathrm{~mm}$, respectively. The variance in $\Delta \mathrm{Y}$ was greater than that in $\Delta \mathrm{X}$, and large uncertainties exist in the determination of the first dwell position. The $95 \%$ confidence limit was $2.1 \mathrm{~mm}$.

Conclusions: In HDR brachytherapy, an effectiveness of independent source position assessment could be demonstrated. The $95 \%$ confidence limit was $2.1 \mathrm{~mm}$ for a tandem-ovoids applicator.

J Contemp Brachytherapy 2017; 9, 5: 477-486 DOI: https://doi.org/10.5114/jcb.2017.70952
\end{abstract}

Key words: external audit, gynecological, source position.

\section{Purpose}

Brachytherapy sources have a distinguishing dose distribution with a high gradient of dose near the source, which can provide radiation oncologists with a high-dose conformity for tumors. However, the dislocation of these sources could cause an unexpectedly high dosimetric impact $[1,2]$. It is therefore important to verify the accuracy of the source position through a routine quality assurance program $[3,4,5,6]$. In addition, inaccurate applicator reconstruction in treatment planning systems (TPSs) would cause improper planned dose distributions with wrong source positions in the TPS. Such an error could not be de- tected by a traditional method using a radiographic film, dedicated to quality assurance tool only for mechanical source position. This is because its procedure does not include the applicator reconstruction process. Therefore, it is necessary to directly compare a dose distribution between the TPS and measurements under the same condition as clinical practice. This is referred to as an "end-to-end test". Several studies reported that such an assessment involves measurements in a condition close to clinical practice, such as film irradiation [7], pinhole cameras [8], two-dimensional detectors [9], and C-arm fluoroscope [10].

Because it is difficult to monitor actual brachytherapy source positions during treatments and a dose per 
fraction in brachytherapy is higher than a conventional dose per fraction of 2 Gy in external radiotherapy, conducting end-to-end test must be essential especially in brachytherapy. However, there are few reports published about the establishment of an end-to-end test, and the audit system continues to be developed for brachytherapy $[7,11,12,13,14,15]$. For instance, the ESTRO (European Society for Radiotherapy \& Oncology) project demonstrated the effectiveness of the postal audit in brachytherapy, and it was found that $14 \%$ of the participating institutions were outside the tolerance level in geometric reconstruction [11]. Awunor et al. also demonstrated the effectiveness of the end-to-end system by irradiation of the film for assessment of source positioning uncertainty for ring applicator [13]. Australasian brachytherapy audit could provide the dosimetry check system using the thermoluminescent dosimeter (TLD), reported by Haworth et al. [14]. Palmer et al. [7,15] established fully end-to-end dosimetry audit for imaging, applicator reconstruction, treatment planning, and delivery. They performed the triple-channel film dosimetry at 46 centers to assess the TG- 43 source data in TPSs, and they concluded that dose distributions in film agreed well with dose distributions according to the TG-43, which can provide accurate film dosimetry for external audit in brachytherapy.

In this study, we focus on all source position uncertainties, which can include display locations of isodose curve, applicator reconstruction techniques, and delivery. That is because operational errors made in the TPS have frequently occurred during recent years in Japan. Then, the need of an external audit system including all processes has been discussed during domestic intersociety meeting. The developed phantom in this study has a unique design and simple mechanism to fix the radiochromic film and an intricately shaped tandem-ovoid applicator. This study was performed to evaluate the discrepancies of a dwell position between TPS and film under the same conditions as those in clinical routine, using the developed phantom. In addition, reproducibility of source transfer and the measurement uncertainty were evaluated. As a trial, the phantom was mailed to 12 radiotherapy institutions, in which the accuracies of an ${ }^{192}$ Ir source transfer were assessed, and the effectiveness of this test was demonstrated.

\section{A}

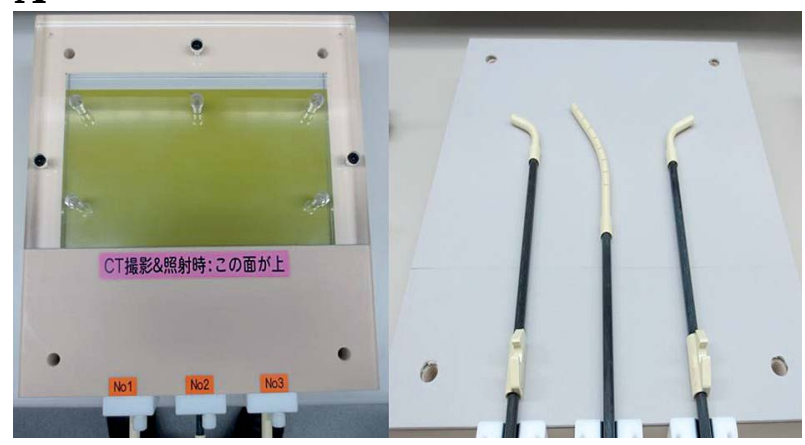

\section{Material and methods}

\section{Phantom design}

Figure 1 shows the developed phantom with a simple mechanism capable of assessment of the source positions between the TPS and the films. Three applicators (or catheters) can be firmly fixed by the jig attached to the lower-slab phantom. The applicator jig is capable of fixing different applicators, e.g., gynecological applicators, metallic interstitial needles, and flexible interstitial needles, made by different vendors, e.g., Nucletron (Nucletron, an Elekta company, Stockholm, Sweden), Varian medical systems (Co., Palo Alto, CA, USA), and Eckert \& Ziegler BEBIG (Berlin, Germany). However, only a tandem-ovoid applicator was used for this analysis. As shown in Figure 1B, a sheet of Gafchromic ${ }^{\mathrm{TM}}$ EBT3 film (Ashland Inc., NJ, USA) was placed above the applicators for determination of the source positions by irradiation. A low resilience flexible polyurethane foam can be used to firmly fix intricately shaped gynecological applicators. The distance between the film surface and the source position was approximately $12 \mathrm{~mm}$ when ovoids with caps were used. For ovoids without caps or for tandems, this distance was approximately $5 \mathrm{~mm}$. Two radio-opaque markers with a diameter of $1 \mathrm{~mm}$ were implanted below the film on both sides in the phantom to match the dimension of the TPS and film.

\section{Procedure and analysis}

This phantom can allow us to assess the source positions through all processes in brachytherapy treatment: CT scan, treatment planning (applicator reconstruction, source activation), data transfer, and irradiation. Computed tomography images (Aqulion ${ }^{\mathrm{TM}} \mathrm{LB}$, Toshiba Medical Systems Corporation, Canon Group, Tokyo, Japan) are first obtained after setting the film and three applicators (two ovoids and one tandem), as shown in Figure 1A. The CT-images are then transferred to the TPS, and source positions and dwell times in each applicator are set after applicator reconstruction with a maximum dose of 2 to 3 Gy to film surface plane. The treatment parameters are then transferred to the console of the remote afterloading system, and the film is irradiated according to the treatment plan. After the irradiation, the exposed

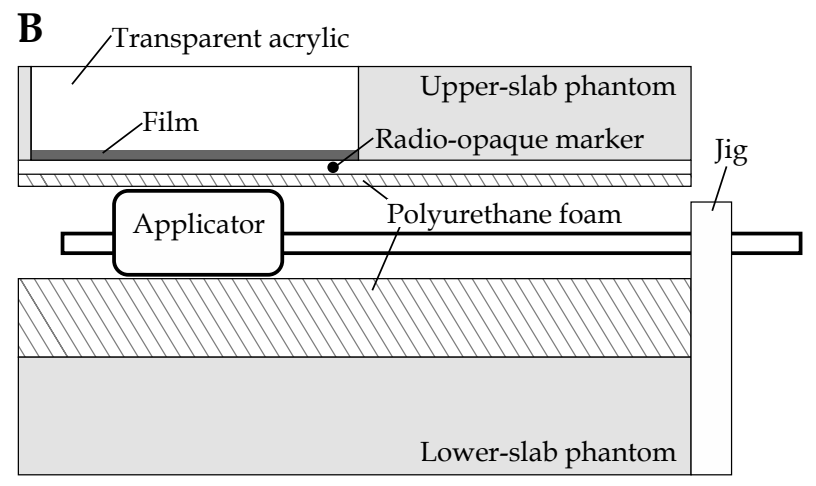

Fig. 1. A) The developed phantom for assessing source positions of the gynecological applicator. The film and two radio-opaque markers are placed above the three applicators. B) The cross-section of the phantom 
film is digitized using an EPSON ES-G11000 flatbed scanner (Seiko Epson Corp., Nagano, Japan) at a resolution of $400 \mathrm{dpi}$, equivalent to $0.064 \mathrm{~mm} /$ pixel, and 16-bit grayscale. The digitized images are analyzed using in-house software built in application development environment of MATLAB version 2015a with the image processing toolbox (the Mathworks, Inc., Natic, MA, USA). As shown in Figure 2A, the position and two lengths of major and minor axis of the ellipse, indicated by a blue arrow in the figure, is manually adjusted to coincide with the isodose curve of 2 to 3 Gy to directly assess a display location of the isodose curve on the coronal plane in the TPS by using the in-house software. For this reason, the DICOM RT-dose was not used. The geometrical center position of the ellipse is defined as the source position in the TPS. Thus, the coronal-view isodose curves of 2 to 3 Gy with a diameter of a few centimeters on the film surface in the TPS are essential as an image file for this analysis. A regression algorithm can be employed for deriving the source position from the optical distribution of the EBT3 film (see Figure 2B). The source positions are determined as the peak locations of two-dimensional Gauss distributions, which can be used as a regression curve for two-dimensional optical distribution with a least-squares method (see Figure 2C).

Figure 3A shows a definition of the source position $P_{\text {TPS }}$ in the TPS and the film source position $P_{\text {Film }}$ in the film. For the definition of $P_{\text {TPS }}$ two captured images from the TPS, which were used to determine the lines $\left(M_{1}\right.$ and $M_{2}$ ) and the transfer angle of the source $\theta$ are prepared to define a new $\mathrm{X}-\mathrm{Y}$ coordinate. The symbol $\theta$ represents the transfer angle of the source in reference to the line between two radio-opaque markers $\left(M_{1}\right.$ and $\left.M_{2}\right)$. The $X-Y$ coordinates are aligned to the direction of the source transfer. The positive direction of the $\mathrm{X}$-axis corresponds to the external side of the applicator perpendicular to the source transfer direction. On the film, the positions of the markers are indicated by dots that are made using a marker pen after the irradiation, and their coordinates were identified by matching the positions of the markers in the acquired CT images of the phantom in the TPS and in the film. Then, the dimensions of the TPS and film are matched by using two radio-opaque markers implanted below the film on both sides in the phantom. By superimposing the two, we obtained the source displacement, as shown in Figure 3B. The origin of the coordinate system is located at the source position determined in the TPS. Therefore, the symbols $\Delta X$ and $\Delta Y$ mean the source displacements in the lateral and longitudinal directions of the source transfer in reference to the TPS position. $\Delta \mathrm{R}$ means the absolute source displacement derived from this calculation of $\sqrt{\Delta X^{2}+\Delta Y^{2}}$. It was emphasized that our in-house software can allow us to obtain source displacements according to the new special coordinate system (Figure 3), by matching the film coordinate to the TPS coordinate (Figure 2).

\section{Dwell source position accuracy}

Three tests were conducted in regard to source position accuracy for the Nucletron Fletcher CT/MR tandem-ovoid applicator and the Nucletron remote afterloading system
A

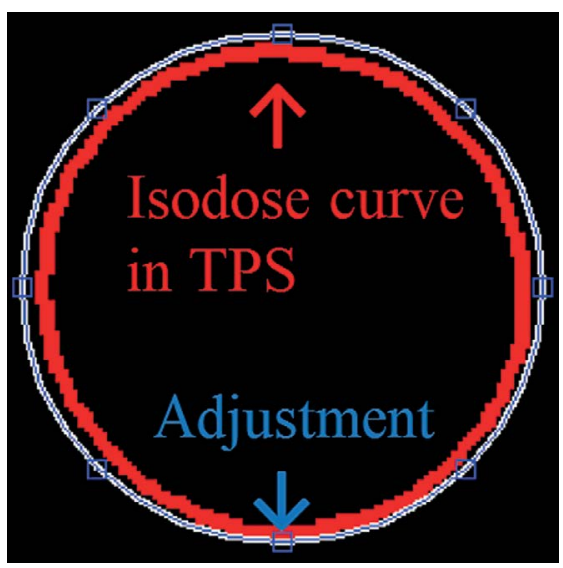

C

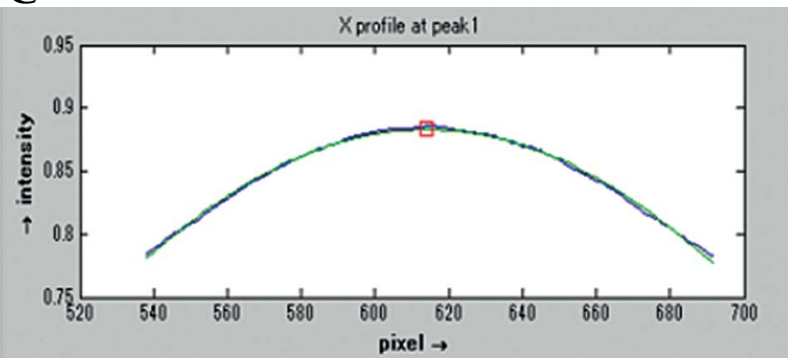

B
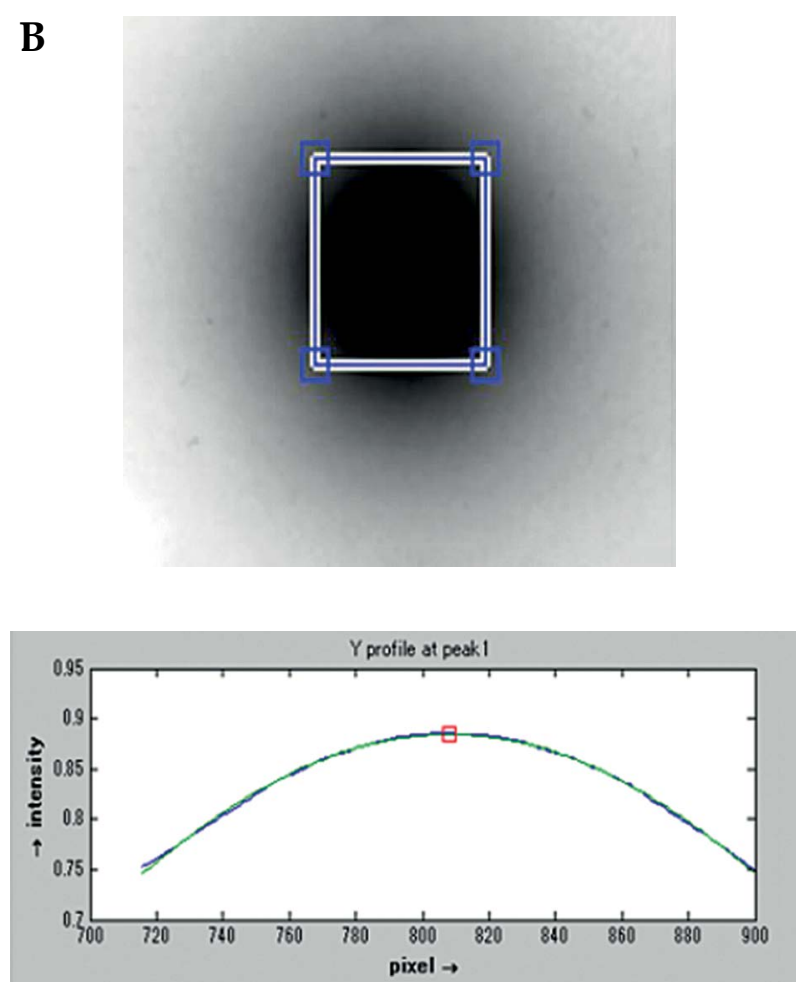

Fig. 2. A) Assessment of a display location of the isodose curve in treatment planning system. B) The source position in the EBT3 film. C) Determination of the source positions as the peak location of two-dimensional Gauss distributions 
A
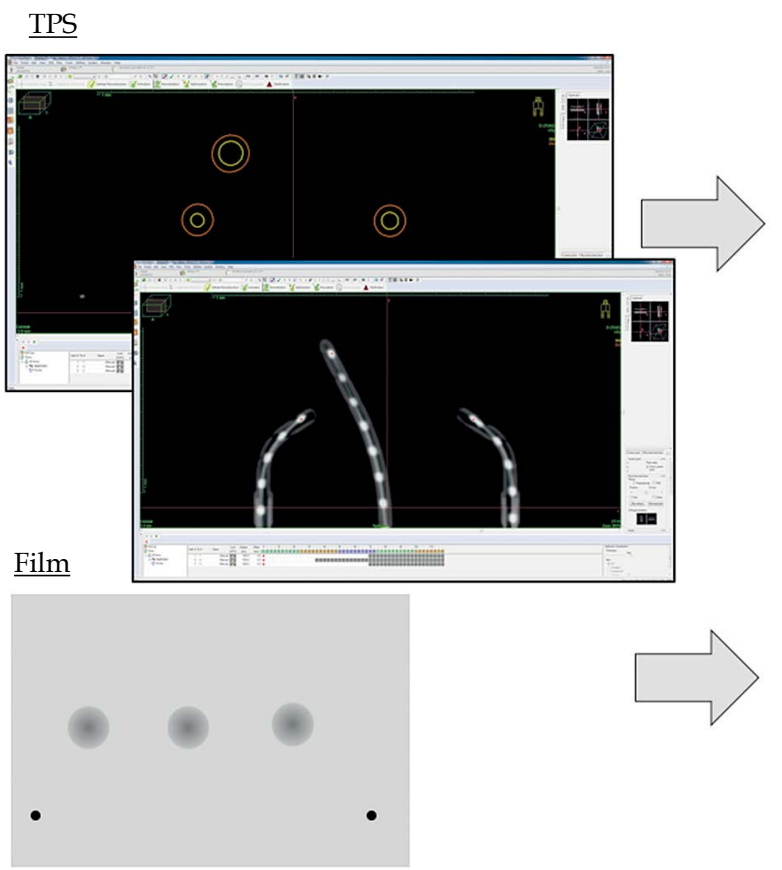

B

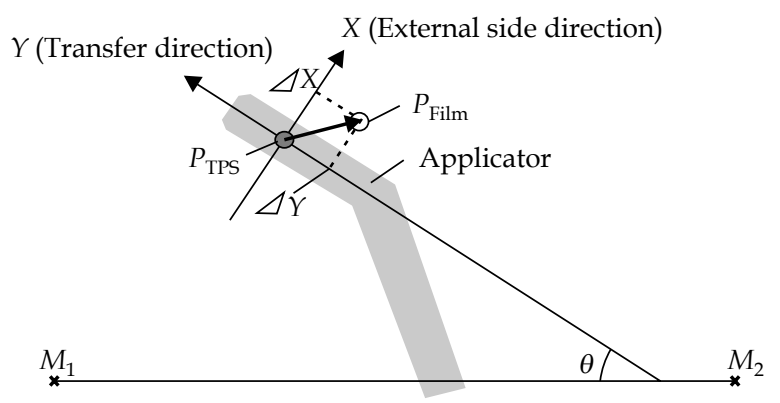

(RALS): 1) reproducibility of source position; 2) a source movement inside the applicator tube; 3 ) influence of source position accuracy by changing curvature of a transfer tube. The details of the three tests are described below.

Test 1: In the developed phantom, relative positions between the applicators and the radio-opaque markers can be fixed when replacing a new film by removal of only the transparent acrylic after the irradiations (see Figure 1B). Therefore, we can repeat the measurements to assess the reproducibility of source positions. In the treatment plan, applicator reconstruction was performed by one medical physicist who defined the central position of the applicator tube on the CT images. The source dwell positions were 0 and $20 \mathrm{~mm}$ from the first dwell position in both left and right ovoid applicator, and 0, 20, 40 , and $60 \mathrm{~mm}$ in tandem (30 degree). The CT slice thickness was $0.5 \mathrm{~mm}$. The irradiations and film measurement were repeated five times with the same reference image including isodose curves from the TPS (i.e., only one TPS

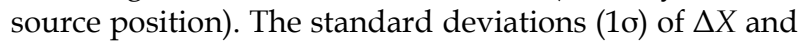
$\Delta Y$ were determined. Test 2: Actual source is pushed toward the outer wall of the applicator by the source cable. It depends on curvature of the applicator. The depen-

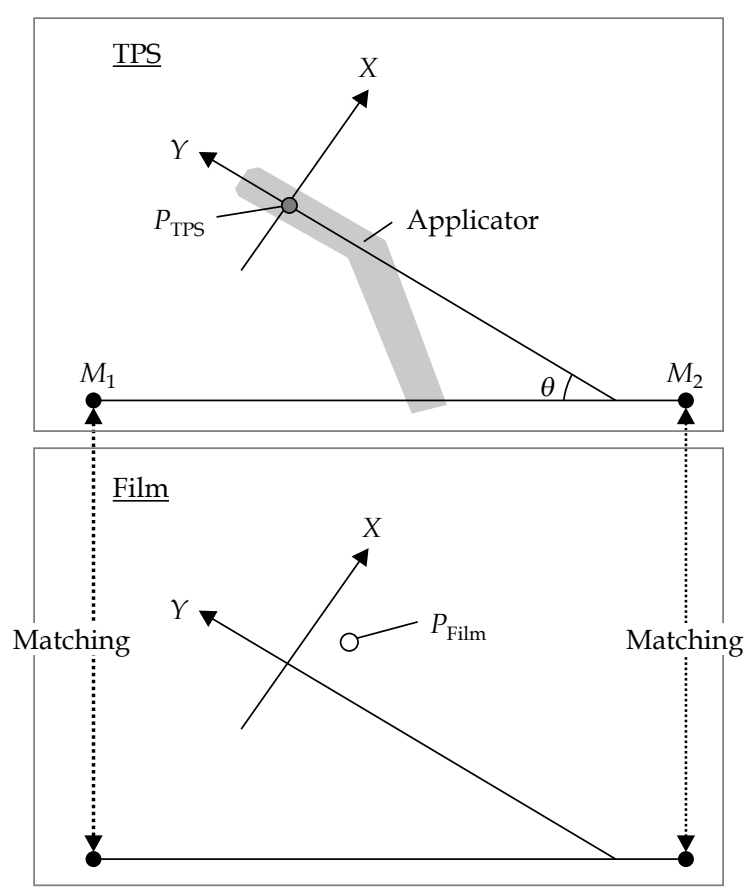

Fig. 3. A) A definition of the source position $P_{\text {TPS }}$ in the treatment planning system and the film source position $P_{\text {Film }}$ in the film. B) The source displacement by superimposing the two. The origin of the coordinate system is located at the $P_{\text {TPS. }}$. The gray color in the figure represents the applicator. The positive direction of the $X$-axis corresponds to the external side of the bending curve of the applicator

dency of $\Delta X$ on the source position was examined for the Nucletron Fletcher CT/MR tandem-ovoid applicator. The inner-diameter of the tandem-ovoid applicator was approximately $3 \mathrm{~mm}$. The source positions from the first dwell position were changed from 0 to $30 \mathrm{~mm}$ and 0 to $80 \mathrm{~mm}$ for the ovoid and tandem applicators, respectively. The bending region for the ovoid and tandem applicator tubes corresponds to 0 to $15 \mathrm{~mm}$ and 0 to $50 \mathrm{~mm}$, respectively. In addition, a metal and a plastic interstitial needle, which have smaller inner-diameters of approximately $1.5 \mathrm{~mm}$ than that of the tandem-ovoid applicator, were verified in this test. In the same way as the tandem-ovoid applicator, the straight interstitial needles were placed on the phantom, and irradiations of the film were performed according to the treatment plan. Test 3 : Figure 4 shows the schematic of the measurements with the four different curvatures of a transfer tube, 0, 90, 180, and 270 degree. In the same way as Test 1 , five irradiations were repeated under each condition and the mean source position was used for this analysis. The source was located at the first dwell position, and the changes in mean source position were derived from the four measurements in reference to the mean source position at 0 degree. 

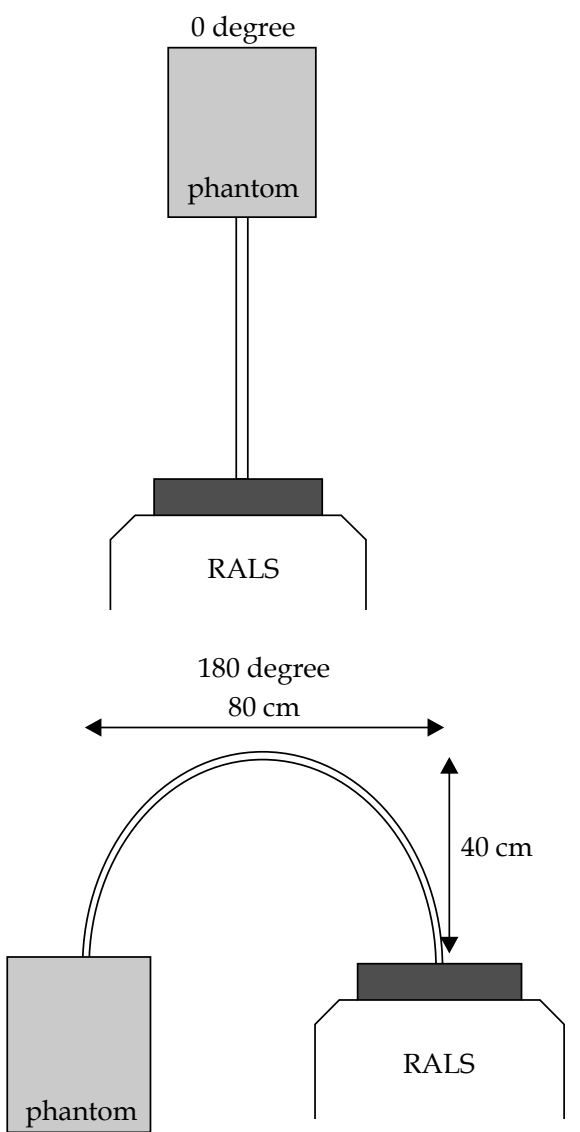

90 degree

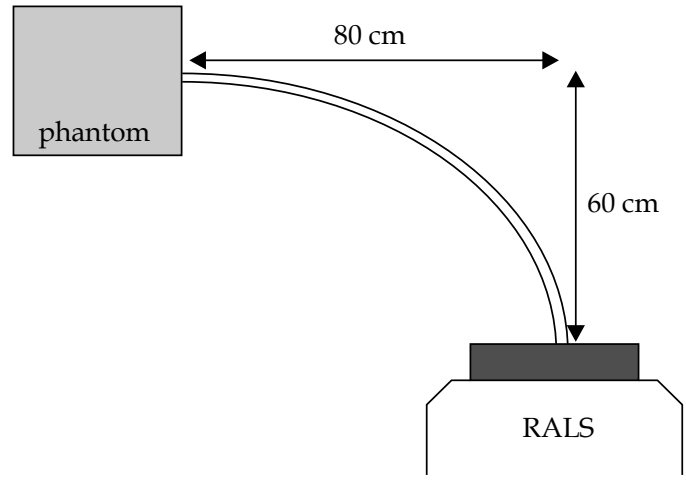

270 degree

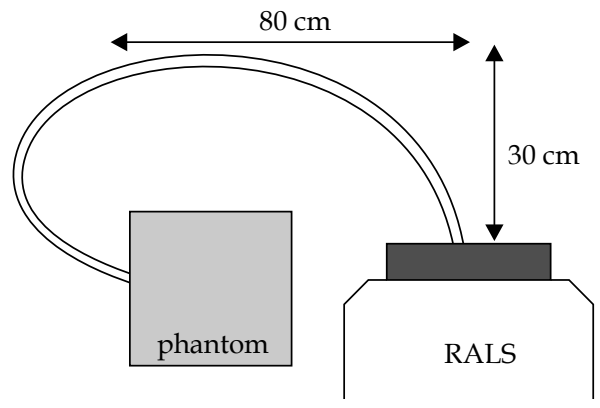

Fig. 4. The geometries of the measurements for the four curvatures of a transfer tube for the Nucletron RALS

\section{Postal audit as a trial study}

We had mailed the developed phantom to 12 radiotherapy institutions (ten Oncentra ${ }^{\circledR}$ Brachy, one BrachyVision $^{\mathrm{TM}}$, and one HDRplus ${ }^{\mathrm{TM}}$ ), and 23 trial data sets were obtained for assessing the source position accuracy to establish external auditing for independent check of the source position in brachytherapy. On average, an institution chose two different conditions, because most institutions tried different methods of applicator reconstruction, e.g., offset, X-ray catheter, or applicator modeling. Table 1 shows four classifications of the 23 trial data sets: applicator, definition of first dwell position, track of the source, and CT slice thickness. The staff at each institution prepared a treatment plan, in which an ${ }^{192}$ Ir source should be placed at the first dwell position in each applicator. After the irradiation, the phantom with the film and some captured images of the treatment plan, as described above were sent to the first author, and staff filled in a dedicated sheet to record treatment planning information such as the applicator reconstruction technique, as described in Table 1.

\section{Results}

\section{Dwell source position accuracy}

Test 1: Figure 5 shows the $1 \sigma$ of the $\Delta X, \Delta Y$ source displacements between five repeated irradiations for the $\mathrm{Nu}$ - cletron RALS. The source displacements were described according to the coordinates shown in Figure 3 (reference $=$ TPS source position). Reproducibility of source posi-

Table 1. Twelve radiotherapy institutions investigated in this study. Number of conducted tests was

23 and mean number of tests by an institute was 2

\begin{tabular}{lc} 
Description & $N$ \\
\hline Category 1: Applicator & 15 \\
\hline CT-MR applicators (15 Nucletron) & 8 \\
\hline Metallic applicators (6 Nucletron, 1 Varian, 1 BEBIG) & \\
\hline Category 2: Definition of 1 $^{\text {st }}$ dwell position & 13 \\
\hline Offset & 7 \\
\hline X-ray catheter & 3 \\
\hline Applicator modeling & 17 \\
\hline Category 3: Track of the source & 3 \\
\hline Defining central position of applicator tube & 3 \\
\hline Defining positions on a X-rays catheter & \\
\hline Applicator modeling & 13 \\
\hline Category 4: Computed tomography slice thickness & \\
\hline$\leq 1.5 \mathrm{~mm}$ &
\end{tabular}


tions $(1 \sigma)$ fell within $1 \mathrm{~mm}$, and the largest $1 \sigma$ of $0.65 \mathrm{~mm}$ in the $X$-axis was observed at the second dwell position in ovoid applicators. Test 2: Figure 6 shows that $\Delta X$ depends on the source position for the Nucletron Fletcher $\mathrm{CT} / \mathrm{MR}$ tandem-ovoid applicator. The source displacements from TPS source positions along the $X$-axis were greater around the tip of the applicator, but these decreased to almost zero around the straight region of the applicator. A maximum source movement of about $1 \mathrm{~mm}$ was observed for both tandem and ovoid applicator components. We applied a linear regression model for each component, which was fitting the data properly. For the metal and plastic interstitial needles, the absolute values of $\Delta X$ were rather small, 0.1 and $0.1 \mathrm{~mm}$, respectively. Test 3: Figure 7 shows the changes of the mean source positions at the first dwell position in reference to the mean source position at 0 degrees for different curvatures of a transfer tube (see Figure 4). The standard deviation in five measurements is shown only for tandem. All of the source position changes fell within $1 \mathrm{~mm}$. At the maximum, the source position change was $0.6 \mathrm{~mm}$ in $\Delta Y$ even for the greatest curvature of 270 degrees.

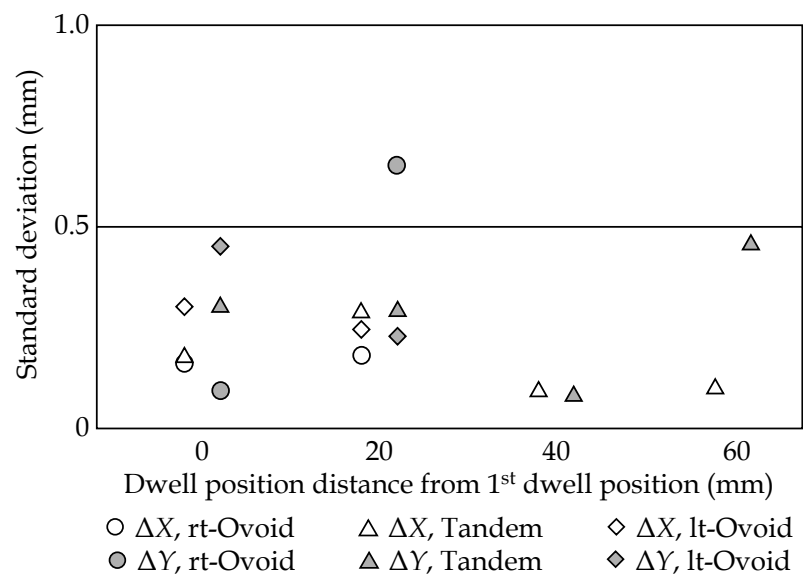

Fig. 5. The $1 \sigma$ in the $\Delta X$ and $\Delta Y$ from five repeated irradiations for the Nucletron RALS

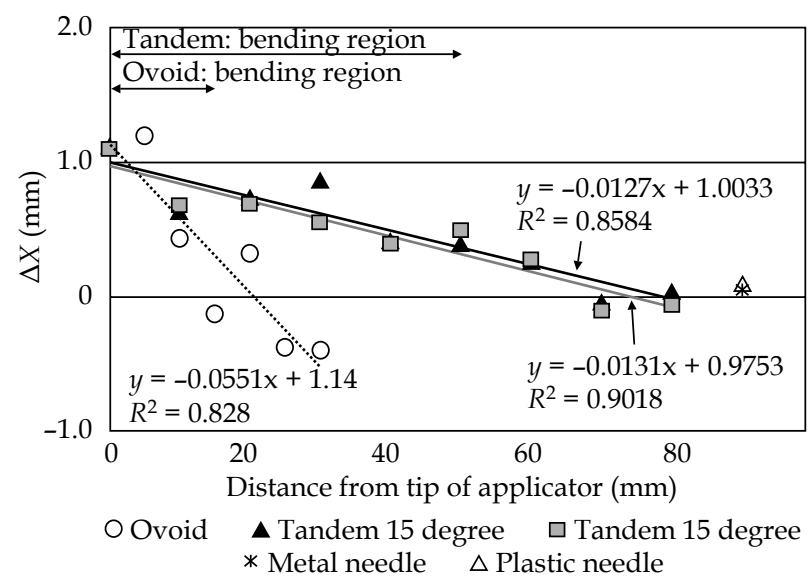

Fig. 6. Dependency of $\Delta X$ on the source position for $\mathrm{Nu}$ cletron Fletcher CT/MR tandem-ovoid applicator

\section{Postal audit as a trial study}

Figure 8A shows the source displacements between the TPS and the film for three applicators in the 12 institutions, expressed in $X-Y$ coordinates (see Figure 3 ). The coordinate origin corresponds to the source position in the TPS, which was defined by the reconstruction methods listed in Table 1. Almost all data were located within a positive region along the horizontal $X$-axis, as mentioned in the result section of Test 2. Figure $8 \mathrm{~B}$ and $8 \mathrm{C}$ shows a distribution of the source displacements in each axis of the applicator, expressed in $X-Y$ coordinates. The two distributions are quite different. The mean values were found to be 0.8 and $0.3 \mathrm{~mm}$ in $\Delta X$ and $\Delta Y$, respectively, and $1.96 \sigma$ values were 0.8 and $1.6 \mathrm{~mm}$, respectively. Table 2 shows reanalysis data for the source displacements according to the Table 1 . Category 3 could not be analyzed, because only the first dwell position was verified.

\section{Measurement uncertainty}

According to the report of the evaluation of measurement data - a guide to the expression of uncertainty in measurement (GUM) [16], measurement uncertainties were categorized. Table 3 shows the overall uncertainties throughout this analysis with the four following components: 1) determination of the dwell position from the film (fitting accuracy of a Gauss distribution); 2) determination of the radio-opaque marker position in the film; 3) determination of the dwell position from the TPS; 4) determination of the radio-opaque marker position in the TPS. The measurement uncertainties in each component were obtained as below: 1) a measurement uncertainty of $0.1 \mathrm{~mm}$ was estimated from a standard deviation obtained by repeated measurements of peak positions for one film; 2) a measurement uncertainty of $0.3 \mathrm{~mm}$ was estimated from the size of the marker; 3 ) a measurement uncertainty of $0.2 \mathrm{~mm}$ was estimated from the width of the isodose curve in the captured image of the TPS, with a mean resolution of $0.16 \mathrm{~mm} /$ pixel from the 12 in-

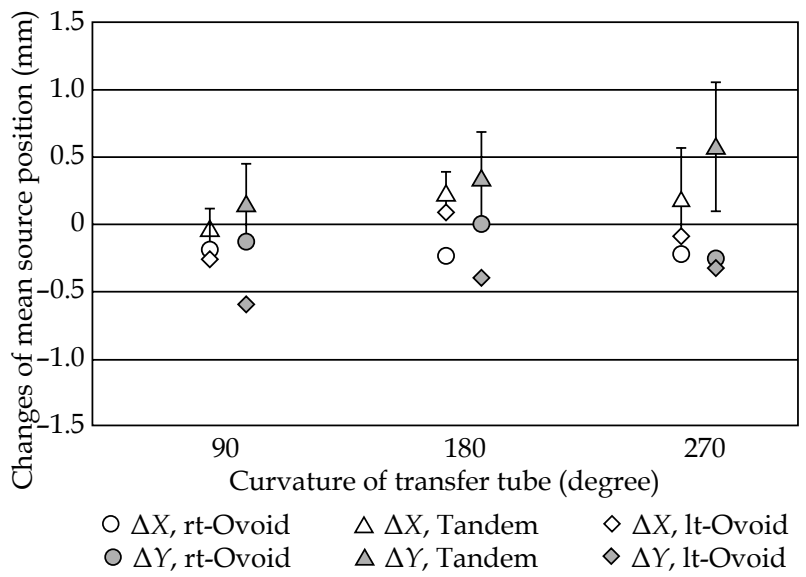

Fig. 7. Influence on source position accuracy of changing the curvature of a transfer tube. Changes in mean source position are shown with reference to the mean source position at 0 degrees 
A

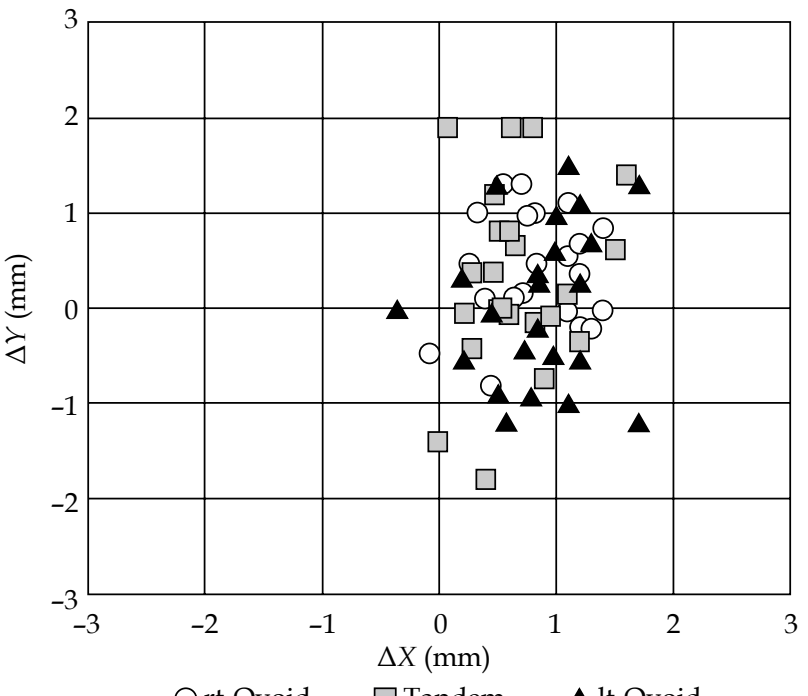

Ort-Ovoid $\square$ Tandem

At-Ovoid

Fig. 8. A) Source displacements between the treatment planning system (TPS) and the film for three applicators. The coordinate origin corresponds to the source position in the TPS. The positive direction of the X-axis corresponds to the external side of the bending curve of the applicator. Distributions of source displacement between the TPS and the film measurements in B) $\Delta X$ and C) $\Delta Y$

stitutions; 4) the measurement uncertainty depends on the slice thickness of the CT images. An uncertainty of $0.5 \mathrm{~mm}$ was estimated by using the profile of the marker in the coronal plane of the CT image with a slice thickness of $3 \mathrm{~mm}$. That is because the maximum slice thickness was $3 \mathrm{~mm}$ in postal audit as a trial study and the spatial resolution of a slice thickness of $3 \mathrm{~mm}$ can be expected to be worst. Finally, an expanded measurement uncertainty of $1.2 \mathrm{~mm}$ could be estimated with the coverage factor $k$ of 2 .

\section{Discussion}

The phantom developed in this study includes the entire treatment procedure and it enables us to check treatment planning processes such as applicator reconstruction, where there are two sub-processes: defining the first dwell position and determining the track of the source. We can choose various applicator reconstruction techniques as described in the Table 1 . However, inaccuracy in both sub-processes leads to unexpected source position uncertainty in the TPS. It is emphasized that applicator reconstruction accuracy should be confirmed by the irradiation of film according to the actual treatment plan, with the applicators used in clinical practice. Conducting a source position check [1] only, as is done in conventional QA/QC program, is insufficient to ensure treatment accuracy.

In Test 1 , it was found that the Nucletron RALS has high precision control of the positioning reproducibility of the ${ }^{192}$ Ir source. It does not depend on the locations of the dwell positions, 0 to $20 \mathrm{~mm}$ in the ovoids and 0 to $60 \mathrm{~mm}$
B

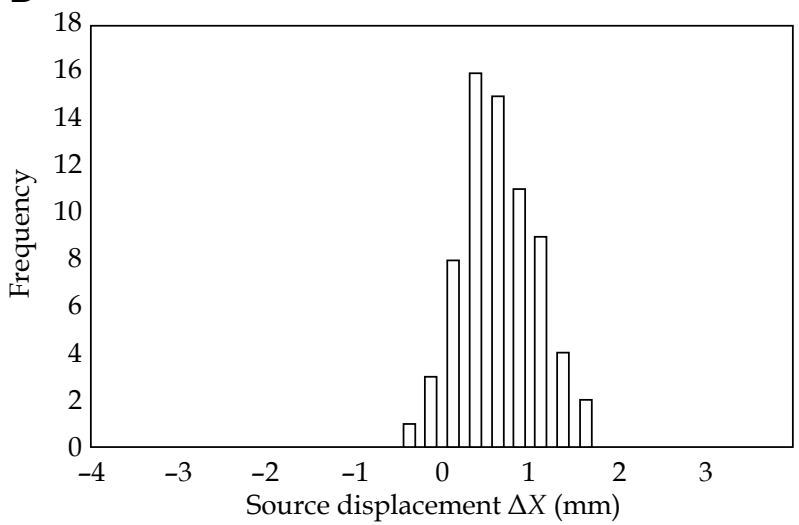

C

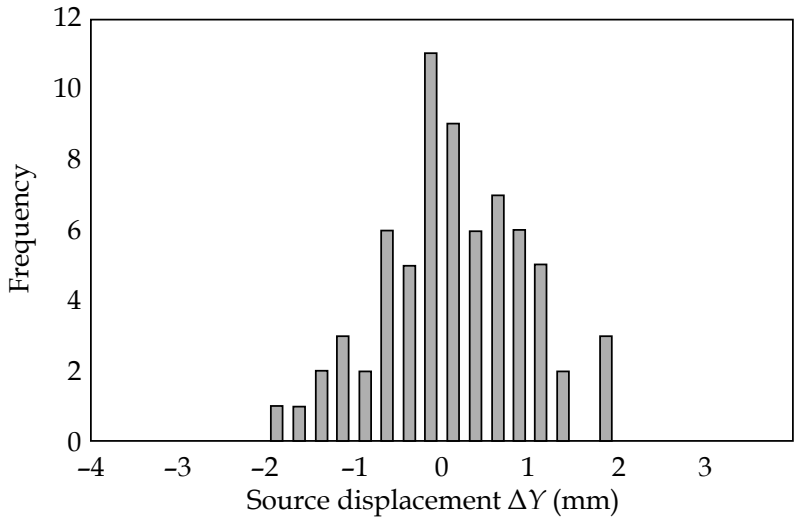

in the tandem. The mean value of $1 \sigma$ was $0.24 \mathrm{~mm}$. A similar reproducibility has been also reported in our previous study, in which a source position accuracy of $0.11 \mathrm{~mm}$ was determined using a conventional QC procedure [1]. Test 2: For source movement along the $X$-axis, the measured source position shifted toward the outer wall of the bending curve of the applicator tube owing to the source cable. A maximum source movement of approximately $1 \mathrm{~mm}$ was observed around the tip of both the tandem and the ovoid applicator components. The tandem-ovoid applicator has an inner-diameter of about $3 \mathrm{~mm}$, and the diameter of the ${ }^{192} \mathrm{Ir}$ source is $0.9 \mathrm{~mm}$. If the source position in the TPS is determined as the central position in the applicator tube, a source movement of approximately $1 \mathrm{~mm}$ potentially occurs, which agrees well with our data. This movement was also described previously $[17,18,19]$. Test 3 : The four different curvatures of a transfer tube were verified to consider clinical situations in practice. The Nucletron RALS does have specifications of the curvature of the transfer tube, and the minimum allowable radius is $10 \mathrm{~cm}$ from a perspective of functioning source transfer (accuracy is not specified) [20]. Although the four radii of the transfer tube were greater than $10 \mathrm{~cm}$ in this study, the influence of changing the curvature radius of a transfer tube was found to be small.

As shown in Figure 8 and Table 2, the postal audit confirmed small displacements along the $X$-axis in the case that the first dwell position was defined by the marker of an X-ray catheter. That is because the first marker 
Table 2. Reanalysis data for the source displacements according to the Table 1 . The unit is $\mathrm{mm}$

\begin{tabular}{|c|c|c|c|c|c|c|c|}
\hline \multirow[t]{2}{*}{ Description } & \multirow[t]{2}{*}{$N$} & \multicolumn{3}{|c|}{ Mean } & \multicolumn{3}{|c|}{$1 \sigma$} \\
\hline & & $\Delta x$ & $\Delta Y$ & $\Delta R$ & $\Delta X$ & $\Delta Y$ & $\Delta R$ \\
\hline \multicolumn{8}{|l|}{ Category 1} \\
\hline CT-MR applicator & 15 & 0.7 & 0.3 & 1.1 & 0.5 & 0.8 & 0.5 \\
\hline Metallic applicator & 8 & 0.8 & 0.1 & 1.2 & 0.4 & 0.9 & 0.4 \\
\hline \multicolumn{8}{|l|}{ Category 2} \\
\hline Offset & 13 & 0.8 & 0.2 & 1.2 & 0.3 & 0.8 & 0.4 \\
\hline $\mathrm{X}$-ray catheter & 7 & 0.3 & 0.1 & 0.7 & 0.4 & 0.7 & 0.5 \\
\hline Applicator modeling & 3 & 0.8 & 0.4 & 1.1 & 0.4 & 0.7 & 0.5 \\
\hline Category 3 & NA & NA & NA & NA & NA & NA & NA \\
\hline \multicolumn{8}{|l|}{ Category 4} \\
\hline$\leq 1.5 \mathrm{~mm}$ & 13 & 0.8 & 0.5 & 1.1 & 0.4 & 0.7 & 0.5 \\
\hline$\leq 3.0 \mathrm{~mm}$ & 10 & 0.8 & 0.0 & 1.2 & 0.5 & 0.9 & 0.5 \\
\hline Total & 23 & 0.8 & 0.3 & 1.1 & 0.4 & 0.8 & 0.5 \\
\hline Confidence limit = & & & & & & & 2.1 \\
\hline
\end{tabular}

CT-MR - computed tomography/magnetic resonance imaging

Table 3. The measurement uncertainty of the developed measurement system in this study. The unit is $\mathrm{mm}$

\begin{tabular}{lc} 
Component & $\begin{array}{c}\text { Measurement } \\
\text { uncertainty }\end{array}$ \\
\hline $\begin{array}{l}\text { (i) Determination of the dwell position from } \\
\text { the film }\end{array}$ & 0.1 \\
\hline $\begin{array}{l}\text { (ii) Determination of the radio-opaque marker } \\
\text { position in the film }\end{array}$ & 0.3 \\
\hline $\begin{array}{l}\text { (iii) Determination of the dwell position from } \\
\text { the TPS }\end{array}$ & 0.2 \\
\hline $\begin{array}{l}\text { (iv) Determination of the radio-opaque marker } \\
\text { position in the TPS }\end{array}$ & 0.5 \\
\hline Combined standard uncertainty & 0.6 \\
\hline $\begin{array}{l}\text { Expanded uncertainty }(k=2) \\
\text { TPS - treatment planning system }\end{array}$ & 1.2
\end{tabular}

location of an X-ray catheter is close to the actual source position. However the coincidence between the two positions is not investigated at other dwell positions. Figure 9 shows the EBT3 film with tandem-ovoid applicators irradiated by a ${ }^{192} \mathrm{Ir}$ source and a $12 \mathrm{MeV}$ electron beam using a conventional linac. By insertion of an X-ray catheter into the applicators for $12 \mathrm{MeV}$ electron irradiation, the markers of the X-ray catheter are visible, and allows us to confirm both the ${ }^{192}$ Ir source positions and the marker positions of the X-ray catheter. As the results, it was seen that the first marker location of the X-ray catheter was close to the actual source position.

The variance of $\Delta Y$ was twice as much as that of $\Delta X$ irrespective of variations in planning technique such as the method of determining the first dwell position in the applicator, and CT slice thickness. The large uncertainty in the determination of the first dwell position depends on the visibility of the X-ray catheters and the applicator on the CT images, and can vary with adjustment of the window/level. For improvement of applicator reconstruction accuracy, the track accuracy of the source in the TPS should be confirmed in a multiple-plane view such as axial, coronal, and sagittal-plane view after the applicator reconstruction.

As shown in Table 2, for the absolute source displacement $\Delta R$, the mean value was $1.1 \mathrm{~mm}$, and 1.960 value was $1.0 \mathrm{~mm}$. The $95 \%$ confidence limit, derived from the sum of the mean and $1.96 \sigma$ values, was found to be $2.1 \mathrm{~mm}$ for the tandem-ovoid applicator in CT-image-guided treatment planning. In this study, we assessed source position uncertainty at only the first dwell position, and the $95 \%$ confidence limit was established from the results. This confidence limit will be used for the tolerance level of actual external audit in brachytherapy, and we will assess multiple dwell positions in each applicator, e.g., 0 and $20 \mathrm{~mm}$ from the first dwell position for ovoid applicator and $0,30,60$, and $90 \mathrm{~mm}$ for tandem. If the result will be beyond the tolerance level, firstly we will ask the institution's staff about irradiation conditions and the treatment planning in detail. If operational errors in TPS or inaccuracy of applicator reconstruction occurs, the new capture image of treatment planning will be analyzed, or the irradiations will be performed again. If the results do not fall within the tolerance level, we will further investigate by visiting the institution to find out the cause.

The developed phantom in this study is available only for tandem-ovoid applicators, not for ring applicators, because the tandem-ovoid applicator in Japan is commonly 

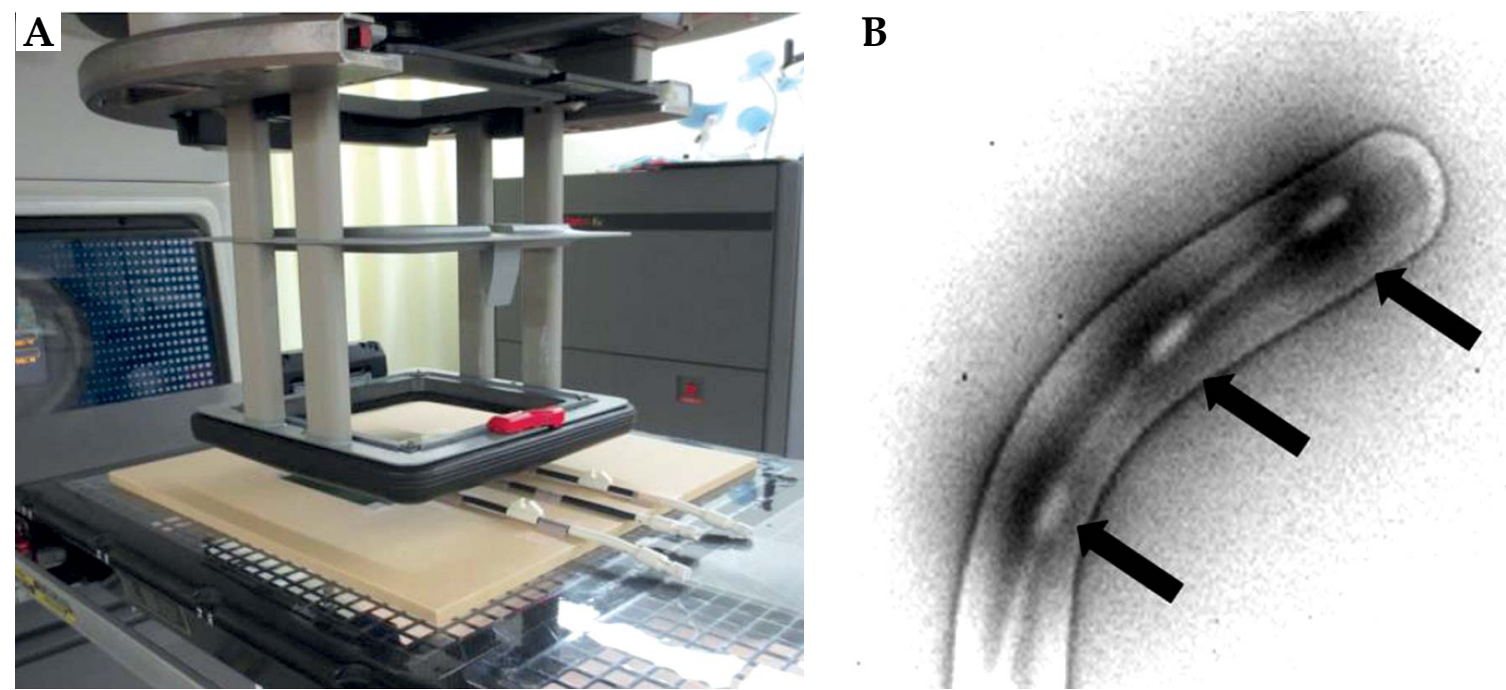

Fig. 9. A) The EBT3 film with tandem-ovoid applicators irradiated by a ${ }^{192} \mathrm{Ir}$ source and a $12 \mathrm{MeV}$ electron beam using a conventional linac. B) The arrow indicates the markers of the X-ray catheter

used rather than the ring applicator. We can only evaluate two-dimensional source displacement on the film plane. Thus, we cannot evaluate three-dimensional source displacement and dosimetric changes arising from source position uncertainty in this study. Moreover, the developed phantom in this study focuses on the source position uncertainty. Thus, it cannot check the source strength independently and assess the dose calculation accuracy in a TPS.

\section{Conclusions}

In this study, we have demonstrated that the developed phantom was effective for independent assessment of the accuracy of transferring the source position from TPS to RALS under the same conditions as those in clinical practice. A measurement uncertainty of $1.2 \mathrm{~mm}$ was obtained in the developed phantom. The $95 \%$ confidence limit of the source displacement was found to be $2.1 \mathrm{~mm}$ for the tandem-ovoid applicator in the prevalent CTimage-guided treatment planning.

\section{Funding information}

This research is partially supported by the Japan Agency for Medical Research and development, AMED (Grant Number 16ck0106039h0002), the National Cancer Center Research and Development Fund (29-E-10), and Grant-in-Aid for Young Scientists (B) from Ministry of Education, Culture, Sports, Science and Technology (Grant Number 26860411).

\section{Acknowledgements}

The authors would like to thank Mr. Sasamori K from Tokyo Medical and Dental University, Kadoya N from Tohoku University Hospital, Kakinohana Y from University of the Ryukyu Hospital, Matsugi K from Shiga Medical Center for adults, Sato $\mathrm{H}$ from Tochigi Cancer Center, Nemoto M from Jichi Medical University, Fujita $Y$ and Kabuki S from Tokai University Hospital, Hirose T from
Kyushu University Hospital, Kinoshita M and Kurokawa $\mathrm{C}$ from Juntendo University Hospital for the technical support and advice about the procedure of the phantom developed in this study.

\section{Disclosure}

Authors report no conflict of interest.

\section{References}

1. Okamoto H, Aikawa A, Wakita A et al. Dose error from deviation of dwell time and source position for high doserate ${ }^{192}$ Ir in remote afterloading system. J Radiat Res 2014; 55: 780-787.

2. Tanderup K, Hellebust TP, Lang S et al. Consequences of random and systematic reconstruction uncertainties in 3D image based brachytherapy in cervical cancer. Radiother Oncol 2008; 89: 156-163.

3. Thomadsen BR, Erickson BA, Eifel PJ et al. A review of safety, quality management, and practice guidelines for high-doserate brachytherapy: Executive summary. Pract Radiat Oncol 2014; 4: 65-70.

4. Kutcher GJ, Coia L, Gillin M et al. Comprehensive QA for Radiation Oncology: Report of AAPM Radiation Therapy Committee Task Group 40. Med Phys 1994; 21: 581-618.

5. Nath R, Anderson LL, Meli JA et al. Code of practice for brachytherapy physics: Report of the AAPM Radiation Therapy Committee Task Group No. 56. Med Phys 1997; 24: 15571598.

6. ESTRO. European Society for Therapeutic Radiology and Oncology. A practical guide to quality control of brachytherapy equipment. European guidelines for quality assurance in radiotherapy, ESTRO Booklet No 8. ESTRO, Brussels 2004.

7. Palmer AL, Lee C, Ratcliffe AJ et al. Design and implementation of a film dosimetry audit tool for comparison of planned and delivered dose distributions in high dose rate (HDR) brachytherapy. Phys Med Biol 2013; 58: 6623-6640.

8. Duan J, Macey D, Pareek P et al. Real-time monitoring and verification of in-vivo high dose rate brachytherapy using a pinhole camera. Med Phys 2001; 28: 167-173.

9. Espinoza A, Petasecca M, Fuduli I et al. The evaluation of a 2D diode array in "magic phantom" for use in high dose 
rate brachytherapy pretreatment quality assurance. Med Phys 2015; 42: 663-673.

10. Nose T, Chatani M, Otani $Y$ et al. Real-Time Verification of a High-Dose-Rate Iridium 192 Source Position Using a Modified C-Arm Fluoroscope. Int J Radiat Oncol Biol Phys 2017; 97: 858-865.

11. Roué A, Ferreira IH, Van Dam JV et al. The EQUAL-ESTRO audit on geometric reconstruction techniques in brachytherapy. Radiother Oncol 2006; 78: 78-83.

12. Elfrink RJM, Kolkman-Deurloo I-KK, van Kleffens HJ et al. Determination of the accuracy of implant reconstruction and dose delivery in brachytherapy in The Netherlands and Belgium. Radiother Oncol 2001; 59: 297-306.

13. Awunor O, Berger D, Kirisits C. A multicenter study to quantify systematic variations and associated uncertainties in source positioning with commonly used HDR afterloaders and ring applicators for the treatment of cervical carcinomas. Med Phys 2015; 42: 4472-4483.

14. Haworth A, Wilfert L, Butler D et al. Australasian brachytherapy audit: Results of the 'end-to-end' dosimetry pilot study. J Med Imaging Radiat Oncol 2013; 57: 490-498.

15. Palmer AL, Diez P, Gandon L et al. A multicentre 'end to end' dosimetry audit for cervix HDR brachytherapy treatment. Radiother Oncol 2015; 114: 264-271.

16. JCGM100: 2008. Evaluation of measurement data-Guide to the expression of uncertainty in measurement. International Organization for Standardization (ISO), Joint Committee for Guides in Metrology. Available at: http://www.bipm.org/ utils/common/documents/jcgm/JCGM_100_2008_E.pdf.

17. Evans MD, Devic S, Podgorsak EB. High dose-rate brachytherapy source position quality assurance using radiochromic film. Med Dosim 2007; 32: 13-15.

18. Berger D, Dimopoulos J, Pötter R et al. Direct reconstruction of the Vienna applicator on MR images. Radiother Oncol 2009; 93: 347-351.

19. Kirisits C, Rivard MJ, Baltas D et al. Review of clinical brachytherapy uncertainties: Analysis guidelines of GECESTRO and the AAPM. Radiother Oncol 2014; 110: 199-212.

20. Transfer Tube Set CT/MR GYN for microSelectron 111.006 User Manual, Elekta. Available at: https:/ / www.elekta.com/ brachytherapy/ 